\title{
Syntactic Processing Modulates the $\theta$ Rhythm of the Human EEG
}

\author{
Marcel C. M. Bastiaansen, *' J os J . A. van Berkum,*'†' and Peter Hagoort*'† \\ *Max Planck Institute for Psycholinguistics, P.O. Box 310, 6500 AH Nijmegen, The Netherlands; †F. C. Donders Centre \\ for Cognitive Neuroimaging, P.O. Box 9101, 6500 HB Nijmegen, The Netherlands; and ¥Department of Psychology, \\ University of Amsterdam, Roetersstraat 15, 1018 WB Amsterdam, The Netherlands
}

Received March 26, 2002

Changes in oscillatory brain dynamics can be studied by means of induced band power (IBP) analyses, which quantify event-related changes in amplitude of frequency-specific EEG rhythms. Such analyses capture EEG phenomena that are not part of traditional event-related potential measures. The present study investigated whether IBP changes in the $\delta, \theta$, and $\alpha$ frequency ranges are sensitive to syntactic violations in sentences. Subjects read sentences that either were correct or contained a syntactic violation. The violations were either grammatical gender agreement violations, where a prenominal adjective was not appropriately inflected for the head noun's gender, or number agreement violations, in which a plural quantifier was combined with a singular head noun. IBP changes of the concurrently measured EEG were computed in five frequency bands of $2-\mathrm{Hz}$ width, individually adjusted on the basis of subjects' $\alpha$ peak, ranging approximately from 2 to $12 \mathrm{~Hz}$. Words constituting a syntactic violation elicited larger increases in $\theta$ power than the same words in a correct sentence context, in an interval of $\mathbf{3 0 0 - 5 0 0 ~ m s ~ a f t e r ~ w o r d ~ o n s e t . ~ O f ~ a l l ~ t h e ~}$ frequency bands studied, this was true for the $\theta$ frequency band only. The scalp topography of this effect was different for different violations: following number violations a left-hemispheric dominance was found, whereas gender violations elicited a righthemisphere dominance of the $\theta$ power increase. Possible interpretations of this effect are considered in closing. 2002 Elsevier Science (USA)

\section{INTRODUCTION}

The measurement of event-related potentials (ERPs) has proved to be fruitful in providing a wealth of finegrained information about the time course of cognitive processing events. This is particularly useful for studying the neural basis of language processing, in which timing and dynamics play an important role. It is therefore not surprising that in the past decades there has been a steady increase in the use of ERPs in study- ing the neurocognitive basis of language processing. For instance, one hotly debated issue in the field of psycholinguistics (see Brown and Hagoort, 2000, for a discussion) is whether domain-specific networks exist for semantics and for syntax (as proposed, e.g., by Hagoort et al., 1999) or whether semantics and syntax are processed by the same network (as proposed, e.g., by McClelland et al., 1989). This issue has initiated a search for ERP components that, in the context of language processing, are differentially sensitive to semantic or syntactic processing. To a certain extent, this search has been successful, in that there are qualitative differences between aspects of the ERP waveform that correlate with semantic processing (N400, Kutas and Hillyard, 1980) and those that correlate with syntactic processing (P600/SPS, Osterhout and Holcomb, 1992; Hagoort et al., 1993; and left anterior negativity, or LAN; Friederici et al., 1996; see Kutas and Van Petten, 1995; Brown and Hagoort, 2000, for reviews).

However, recent years have seen an increasing interest in another aspect of EEG and MEG activity, on which we will focus in this paper. This aspect is related to patterns of synchronization and desynchronization that are observed in the oscillatory firing of neurons throughout the brain. Interest in these phenomena has developed as a result of the view that they might provide a window on the processes by which (spatially distributed) brain areas become linked together in dynamic networks (e.g., Singer, 1993). Such distributed network formation has been proposed to be involved in a variety of cognitive operations, e.g., in sensory integration (e.g., Fries et al., 2001), object representation (e.g., Martin and Chao, 2001), memory encoding/ retrieval (e.g., Treves and Rolls, 1994), and possibly also language comprehension (Bastiaansen \& al., 2002).

The voltage or magnetic field changes that are recorded with EEG or MEG sensors are the result of the spatial summation of the activity of a large number of neurons. If groups of neurons, the activities of which are picked up by one and the same sensor, become 
synchronized, this will result in larger EEG/MEG amplitudes for that sensor (e.g., Nunez, 1995). It follows that local changes in synchrony of oscillatory firing patterns lead to changes in amplitude of rhythmic EEG activity. This makes the study of the event-related amplitude changes of frequency-specific EEG/MEG rhythms meaningful. On the other hand, changes in synchronization and desynchronization of oscillatory firing patterns between spatially distant neuronal populations lead to changes in coherence between two (or more) concurrently measured EEG or MEG signals. Thus, the event-related reactivity of either amplitude or coherence of EEG/MEG rhythms is the parameter of interest if one wants to study the local and long-range interactions between neuronal populations, respectively. Below we will point out how the analysis of brain rhythmicity differs from standard ERP methodology.

In order to appreciate the difference between ERP analyses and analyses of the event-related reactivity of EEG/MEG rhythms, it is important to distinguish between two types of activity in the EEG or MEG: induced activity and evoked activity (see also TallonBaudry and Bertrand, 1999). Induced activity is largely rhythmic (oscillatory) in nature and is best considered rhythmic background activity that is modulated, rather than evoked, by experimental events (e.g., Lopes da Silva and Pfurtscheller, 1999). A wellknown example of an induced rhythmic response is the amplitude increase of the occipital $\alpha$ rhythm by the closing of the eyes (Berger, 1929). Note that an important property of induced activity is that it has a fixed temporal relation to an event ( $\alpha$ amplitude increase is temporally contingent upon closing the eyes) without being necessarily phase-locked to the event (closing the eyes always leads to $\alpha$ enhancement, regardless of the phase of the $\alpha$ oscillation at the moment of eye closure). Evoked activity, on the other hand, may be defined as activity that is directly driven by stimuli or state changes. This activity doesn't have to be rhythmic in nature. A well-known example of evoked activity is the N1/P2 complex elicited by the presentation of auditory stimuli. An important property of evoked activity is that it is both time and phase-locked to the event (one and the same stimulus in principle elicits exactly the same waveform each time it is presented).

In ERP research, it is common practice to average EEG or MEG signals over a number of experimental epochs (trials) in order to improve the signal-to-noise ratio. This averaging procedure destroys any temporal structure in the EEG or MEG signal that is not strictly time and phase-locked to the experimental event in question. This is not a problem for studying evoked activity, since this activity is time and phase-locked to the event. For induced activity, the story is different, because the phase of an oscillation is not necessarily the same for each and every epoch. As a result, the induced activity is averaged out of the ERP.

The implication here is that the event-related reactivity of EEG/MEG rhythms cannot be studied with standard ERP methodology. In recent years, tools have been devel oped both for studying amplitude changes of EEG/MEG rhythms (for instance, wavelet analysis, e.g., Tallon-Baudry and Bertrand, 1999, and induced band power (IBP) analysis, e.g., Klimesch, 1999) and for studying coherence changes of EEG/MEG rhythms (event-related coherence analysis, e.g., Nunez et al., 1997, and phase locking statistics, e.g., Lachaux et al., 1999, 2000).

We noted that the study of the neurocognitive basis of language processing can greatly benefit from the use of electrophysiological measures. However, whereas much attention has been devoted to identifying relations between ERPs and different aspects of language processing (see, for example, the reviews in Brown and Hagoort, 1999), the relation between language and oscillatory EEG/MEG dynamics has hardly, if at all, been addressed. In a previous study (Bastiaansen et al., 2002), we investigated for the first time the eventrelated changes in amplitude of two EEG rhythms, the $\alpha$ and $\theta$ rhythms, during language processing. The stimulus material consisted of correct Dutch sentences, which were presented word by word in the visual modality. We found that following each word, an increase in $\theta$ power co-occurred with a decrease in $\alpha$ power, with both effects occurring roughly in the interval of 300$500 \mathrm{~ms}$ after word onset. We proposed that a possible candidate process underlying the phasic $\theta$ increase following individual words in a sentence could be the synchronous activation of a neuronal population corresponding to some aspect of the processing of the word, either in isolation or in its sentence or discourse context. In addition to these phasic word level effects, $\theta$ power increased linearly over the sentence at bilateral temporal, as well as central, electrode positions, whereas $\alpha$ power did not show a linear trend. Since the $\theta$ rhythm has been related to episodic memory processes (e.g., Klimesch, 1999; Kahana et al., 1999; Lisman and Idiart, 1995), we proposed that this gradual $\theta$ power increase may possibly reflect the formation of an episodic memory trace as the individual words in a sentence gradually converge into an overall understanding of the "episode" described by the sentence.

Although these interpretations are rather speculative in nature, the results of this preliminary study do show unequivocally that sentence processing results in reliable, consistent, and highly frequency-specific changes in power in the $\alpha$ and $\theta$ frequency bands. It is the aim of the present paper to investigate to what extent the amplitudes of the $\alpha$ and $\theta$ rhythms are sensitive to the syntactic parsing operations that occur 
during sentence processing. In addition, the $\delta$ band will be studied. To this end, we used stimulus material which consists either of correct Dutch sentences or of Dutch sentences containing syntactic violations. One type of violation involved the agreement of grammatical gender; the other type of violation involved number agreement (see Method).

We used grammatical number and grammatical gender agreement violations for several reasons. First, both types of violations have been shown to elicit robust effects in ERP measures (e.g., see van Berkum et al., 1999a,b; Gunter et al., 2000; Hagoort and Brown, 1999; for gender-related ERP effects, and Hagoort and Brown, 2000; Hagoort et al., 1993; and Osterhout and Mobley, 1995; for number-related ERP effects), a finding that was confirmed by ERP analyses of the present EEG data (van Berkum et al ., 2000). Second, the use of two closely related but not identical types of syntactic violations allowed us to not only explore the reactivity of the $\delta, \theta$, and $\alpha$ rhythms to syntactic violations in general, but also to simultaneously assess their sensitivity to a relatively subtle linguistic distinction.

\section{METHOD}

\section{Subjects}

For the main ERP experiment, 18 right-handed native speakers of Dutch (5 males, age range 19-25, mean age 21) participated in the experiment. None had any neurological impairment, had experienced any neurological trauma, or had used neuroleptics. Also, none of them had participated in the pretest (see below). All subjects were paid a small fee for their participation.

\section{Stimulus Material}

The critical itemset contained 180 Dutch sentence triplets (as in the example given below), each consisting of a correct control sentence, a derived variant that included a gender violation, and a derived variant including a number agreement violation. All correct control sentences were semantically coherent and grammatically correct and contained a predesignated critical singular noun (in boldface in the example), preceded by the Dutch indefinite article een and a prenominal adjective (e.g., donkere in a) of the example). In half of the 180 correct control sentences the critical noun was of common gender (marked COM in the example); in the other half it had neuter gender (marked NEU in the example). In all correct control sentences, the prenominal adjective was appropriately inflected for the head noun's gender, with adjectives modifying a common gender noun correctly taking an -e inflection (e.g., donkere, prachtige) and adjectives modifying a neuter gender noun correctly taking a "zero" inflection (e.g., donker, prachtig). (a) Correct control:

(b) Gender violation:

(c) Number violation:
Ik zag een donkere wolk aan de horizon.

I saw a dark сом $_{\text {cloud }}$ сом on the

Ik zag een donker wolk* aan de horizon.

I saw a dark NEU $_{\text {cloud }}$ COM $*$ on the horizon.

Ik zag enkele donkere wolk* aan de horizon.

I saw several dark cloud* on the horizon. horizon.

For each triplet, the gender violation sentence was derived from the correct control sentence by changing the inflection on the prenominal adjective to that for the alternative gender.

The number violation sentence was derived from the correct control sentence by replacing the indefinite singular article een by a plural quantifier (e.g., Dutch equivalents of two, three, several, various, some, etc.) and by, if necessary, adjusting the adjective inflection to the appropriate -e inflection for plural nouns. Note that apart from the adjective inflection or plural quantifier, the two violation sentences are identical to the corresponding correct control sentence and that, in each, the violation becomes apparent at the same critical singular noun ("wolk"). For number violation sentences, the quantifier was chosen such that if the head noun were to be changed to the plural as well, the resulting sentence would conceptually be as plausible as the corresponding correct control sentence (avoiding noun phrases such as threeeyes, but al so sentences like He grabbed two umbrellas and walked on.). I n order to avoid the possibility that subjects would expect a syntactic violation whenever a plural quantifier was encountered, the stimulus material also contained 60 filler sentences (in addition to 180 unrelated fillers; see the section on design and procedure) in which a plural quantifier was followed by a plural noun. Every sentence contained 12 words at most (8.9 on average), with every word containing at most 12 letters. Critical nouns were constrained to have at most 10 letters (6.1 on average), and they had an average singular word form frequency of 48 on a million in the Dutch $42-$ million-word INL/CELEX corpus. The critical noun phrase was never in sentence-initial position and was followed by at least 2 more words before the sentence's end.

\section{Pretest}

We conducted a paper-and-pencil sentence completion pretest on all 540 critical sentence variants to ascertain that the three types of sentences did not 
differ in the degree of constraint imposed by the sentence context up to the critical noun. Sixty native speakers of Dutch were asked to complete truncated sentence fragments such as Ik zag een donkere (truncated correct control sentence), I k zag een donker (truncated gender violation sentence), or Ik zag diverse donkere ___ (truncated number violation sentence) in a grammatically and conceptually coherent way. Every subject saw 60 truncated sentences of each type, but never saw more than 1 sentence from the same triplet.

F or every truncated sentence, we identified the most frequently generated noun and computed the percentage of subjects that had continued the sentence with the latter (occasionally the target noun, but frequently some other noun). This quantified degree of sentence fragment constraint is of relevance because large differences in this measure are known to affect the amplitude of the N400 elicited by the subsequently presented word (which would in the ERP experiment be our critical noun). The degree of sentence fragment constraint was $36.9 \%$ (SD 19.9) for truncated correct control sentences, 31.0\% (SD 17.5) for truncated gender violation sentences, and 35.9\% (SD 19.5) for truncated number violation sentences, indicating that there were no large differences among conditions in the degree of constraint at the critical word.

F or every truncated sentence, we also computed the percentage of subjects that had continued the sentence with the so-called target noun, i.e., the singular noun that would be presented in the ERP experiment. As expected, this target noun was almost never generated in response to truncated gender $(0.7 \%$, SD 2.5$)$ or number $(0.1 \%$, SD 0.6$)$ violation sentences, whereas truncated correct control sentences elicited the target noun more often (15.1\%, SD 23.0). The difference in cloze probability of the target noun confirms that in this off-line test native speakers of Dutch are sensitive to the syntactic (and, for number, possibly also conceptual) constraints imposed by the preceding sentence context.

\section{Design and Procedure}

In the EEG experiment, every subject saw 60 sentences with a gender violation, 60 sentences with a number violation, and 60 correct control sentences. We created three different trial lists by rotating conditions over items, such that any individual subject saw only one variant of any one triplet. These trial lists were derived from a single randomization, created by pseudorandomly mixing the 180 critical items with 180 unrelated filler items and 60 filler items used to avoid possible expectancies about number violations. The trial lists were constructed such that the largest se-

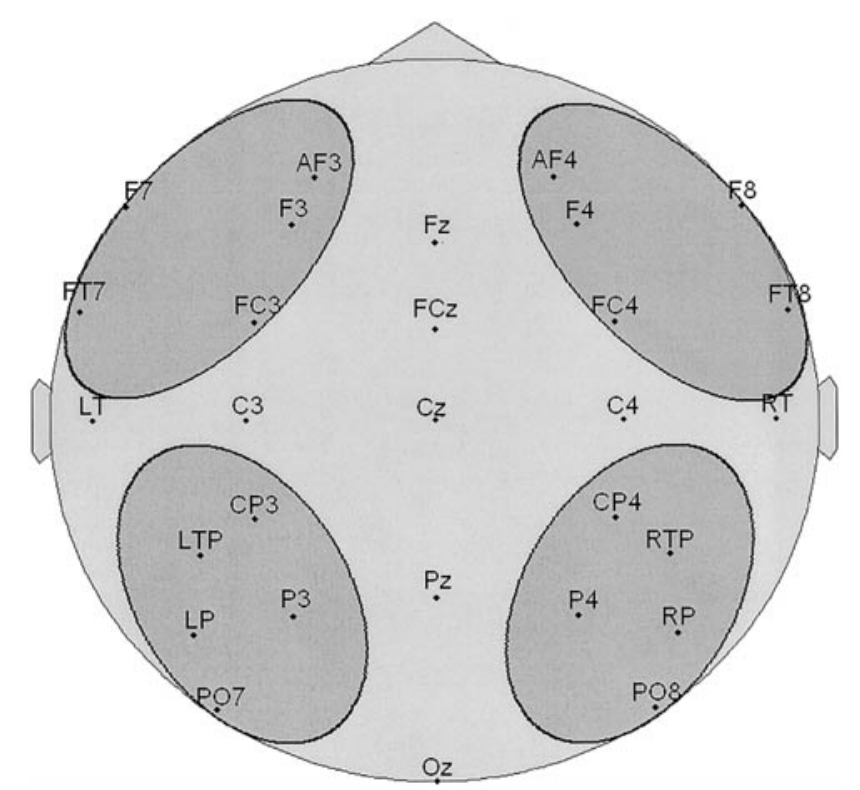

FIG. 1. Orthogonal projection of the electrode configuration. Dark gray areas indicate electrodes used in forming the four quadrants used in the statistical analysis.

quence of consecutive critical trials was 4 and the largest sequence of critical trials in the same condition (e.g., gender violation) was 2 . The trial lists were split up in five blocks of 84 trials, each block starting with at least two filler trials. A 30-trial comparable practice sequence preceded the first experimental block.

After electrode application, subjects were seated in a sound-attenuating booth and asked to silently read every sentence displayed on a computer screen before them. Subjects were told that EEG recording would occur as they read each sentence and that during recording they should avoid all movement, including eye movements and blinks. They were free to move and blink in between trials, whenever an asterisk was displayed on the screen. No additional task demands were imposed.

To avoid eye movements, sentences were displayed word by word at a fixed rate, with every word shown in the center of the screen (serial visual presentation). Each trial consisted of a 300-ms auditory warning tone, followed by a 650-ms blank screen, after which the sentence was presented, with every word displayed for $300 \mathrm{~ms}$ and a 300-ms blank screen between words. To inform subjects when they were free to move and blink, an asterisk was displayed for 2500 ms after every trial, starting $1300 \mathrm{~ms}$ after offset of the sentence-final word. The last $300 \mathrm{~ms}$ of this asterisk display coincided with the warning tone for the next trial. After a short practice, the trials were presented in five blocks of about 14 min, separated by rest periods. 


\section{delta}
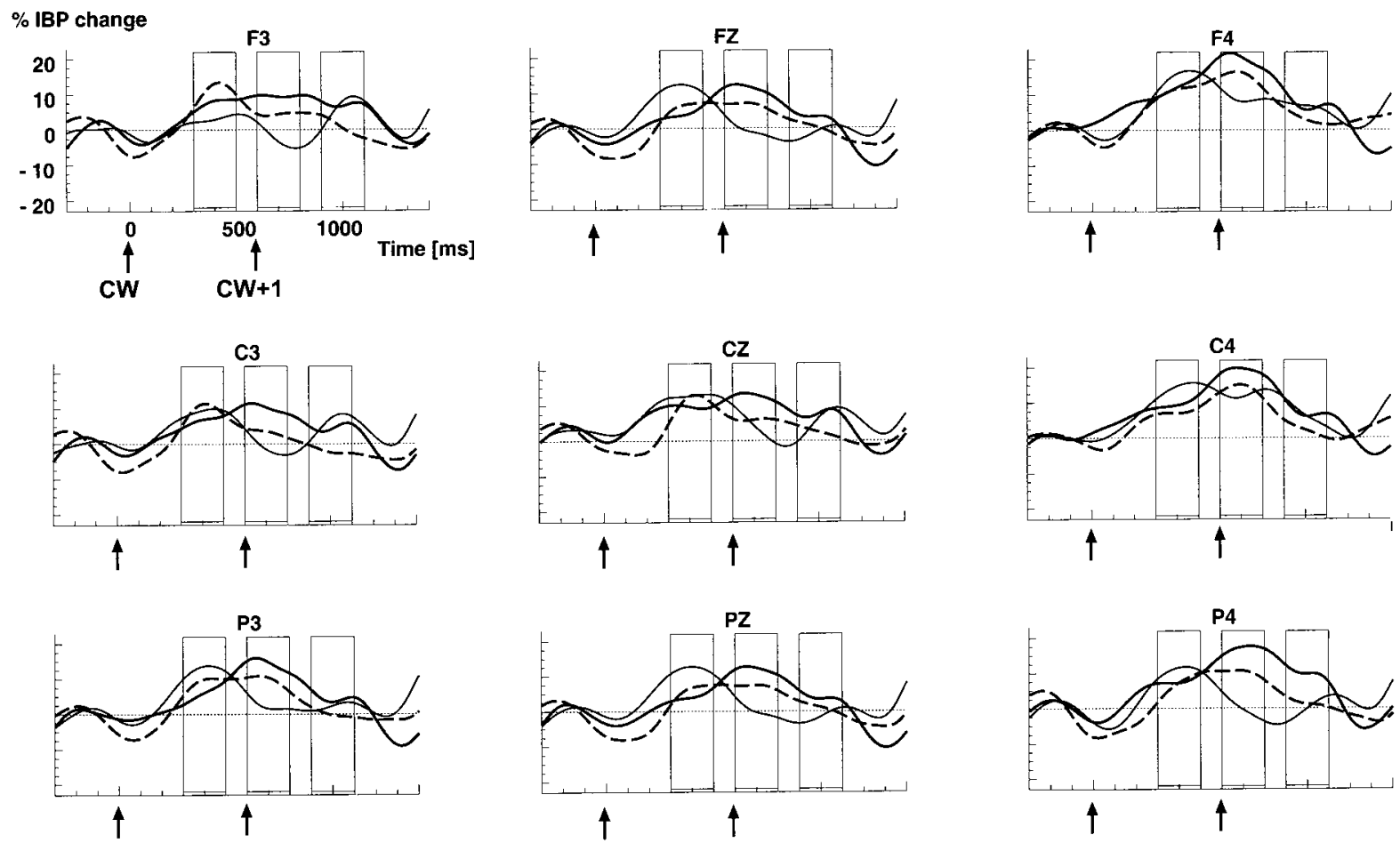

FIG. 2. Time course of IBP changes in the $\delta$ frequency band, at selected electrodes. Black arrows indicate word onsets. CW, critical word. $\mathrm{CW}+1$, word following critical word. The vertical bar corresponds to $=0$, the onset of the critical word. The three rectangular boxes indicate the time intervals used for the statistical analysis.

\section{EEG Recordings}

The EEG was recorded from $29 \mathrm{Ag}-\mathrm{AgCl}$ electrodes mounted in an elastic cap, each referred to the left mastoid. Standard (10\% system) electrode positions were AF3, AF4, F7, F3, Fz, F4, F8, FT7, FC3, FCz, FC4, FT8, C3, Cz, C4, CP3, CP4, P3, Pz, P4, PO7, PO8, and Oz. Nonstandard positions were (a) a temporal pair (LT and RT) placed laterally to Cz, at 33\% of the interaural distance, (b) a temporoparietal pair (LTP and RTP) placed at $30 \%$ of the interaural distance lateral and at $13 \%$ of the nasion-inion distance posterior to $\mathrm{Cz}$, and (C) a parietal pair halfway between LTP/RTP and PO7/PO8 (LP and RP). The electrode configuration is displayed in Fig. 1. Vertical eye movements were monitored via a supra- to suborbital bipolar montage. A right to left canthal bipolar montage was used to monitor horizontal eye movements.

The EEG and EOG recordings were amplified with a NeuroScan SynAmp Model 5083 EEG amplifier, using a hi-cut of $30 \mathrm{~Hz}$ (with an additional notch filter at 60 $\mathrm{Hz}$ ) and a time constant of $8 \mathrm{~s}$. Electrode impedance was kept below $3 \mathrm{kOhm}$ for the EEG recording and below $5 \mathrm{kOhm}$ for the EOG recording. The EEG and EOG signals were digitized on-line with a sampling frequency of $200 \mathrm{~Hz}$ and screened off-line for eye movements, muscle artifacts, electrode drifting, and amplifier blocking in a critical window that ranged from 150 ms before onset of the prenominal adjective to $1600 \mathrm{~ms}$ after onset of the critical noun (i.e., $1300 \mathrm{~ms}$ after its offset). Trials containing such artifacts were excluded from further analysis (10.0\% on average).

\section{IBP Computation}

For the computation of the IBP changes, we followed the approach outlined by Klimesch (1999, see the description of this procedure bel ow). In this approach, the IBP is computed in individually adjusted, narrow (i.e., $2 \mathrm{~Hz}$ ) frequency bands, based on the individual $\alpha$ peak frequency (IAF). The IAF ( $M=10.27 \mathrm{~Hz}, \mathrm{SD}=0.813$ ) was determined for each subject separately, by averaging (both over epochs and over electrodes) the power spectra computed in a 2350-ms window ranging from 750 before to $1600 \mathrm{~ms}$ after the onset of the critical word. 


\section{theta}
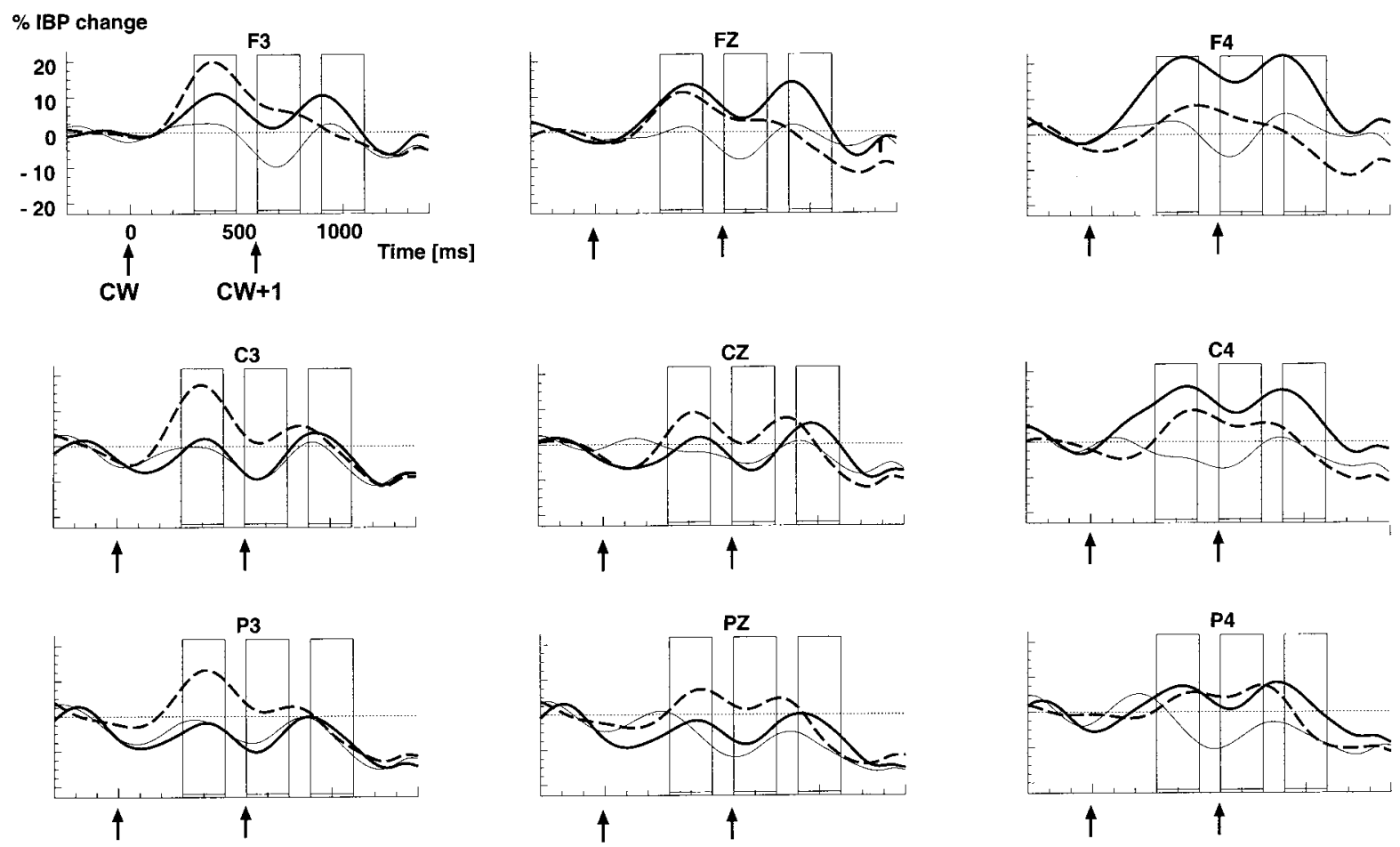

FIG. 3. Time course of IBP changes in the $\theta$ frequency band, at selected electrodes. See legend to Fig. 2 for further details.

The EEG data (from $300 \mathrm{~ms}$ preceding to $1300 \mathrm{~ms}$ following the onset of the CW) were filtered with an FFT-based filter in five different frequency bands defined as follows: $\delta$ (IAF $-8 \mathrm{~Hz}$ to IAF $-6 \mathrm{~Hz}$ ), $\theta$ (IAF $6 \mathrm{~Hz}$ to IAF $-4 \mathrm{~Hz})$, lower-1 $\alpha$ (IAF $-4 \mathrm{~Hz}$ to IAF $2 \mathrm{~Hz}$ ), lower-2 $\alpha$ (IAF $-2 \mathrm{~Hz}$ to IAF), and upper $\alpha$ (IAF to IAF $+2 \mathrm{~Hz}$ ). Next, for each frequency band the data were averaged, and the resulting (filtered) ERP was subtracted from each (filtered) single trial in order to avoid a contamination of the IBP measures with the ERPs (see Kalcher and Pfurtscheller, 1995, for a detailed description of this method and its rationale). On the single-trial data thus obtained, we applied a Hilbert transform with subsequent demodulation, which yields the envelope of the signal. Subsequently the single-trial signal envelopes were averaged over trials, and IBP was computed as the percentage power increase or decrease in a particular frequency band, relative to a reference interval from 300 to $0 \mathrm{~ms}$ preceding the onset of the CW. Note that inherently IBP measures have a temporal resolution that is reduced compared to traditional ERP measures. Elsewhere (Knosche and Bastiaansen, 2002) we investigated the temporal resolution of IBP measures in detail by means of a simulation study. For the present purpose, it is of interest to note that the temporal resolution roughly ranges from 150 to $200 \mathrm{~ms}$ for the $\delta$ and $\theta$ bands and from 100 to 150 ms for the $\alpha$ subbands under investigation. Thus, the temporal resolution should be sufficient (although not ideal) to study processes involved in sentence comprehension. Percentages IBP change were subsequently entered into the statistical analyses, and finally the IBP changes were averaged over all subjects for display purposes only.

\section{Statistical Analyses}

For the statistical analyses of the IBP changes, the electrodes were grouped into four quadrants, each including five electrodes (see also Fig. 1): left anterior, including electrodes AF3, F3, F7, FC3, and FT7; left posterior, including electrodes LTP, CP3, P3, LP, and PO7; right anterior and right posterior, including the homologous electrodes over the right hemisphere.

Three time windows were selected for analysis, partly on the basis of the results of the ERP analyses on the same data (Van Berkum et al., 2000) and partly dictated by the results of a previous study on IBP 


\section{lower-1 alpha}
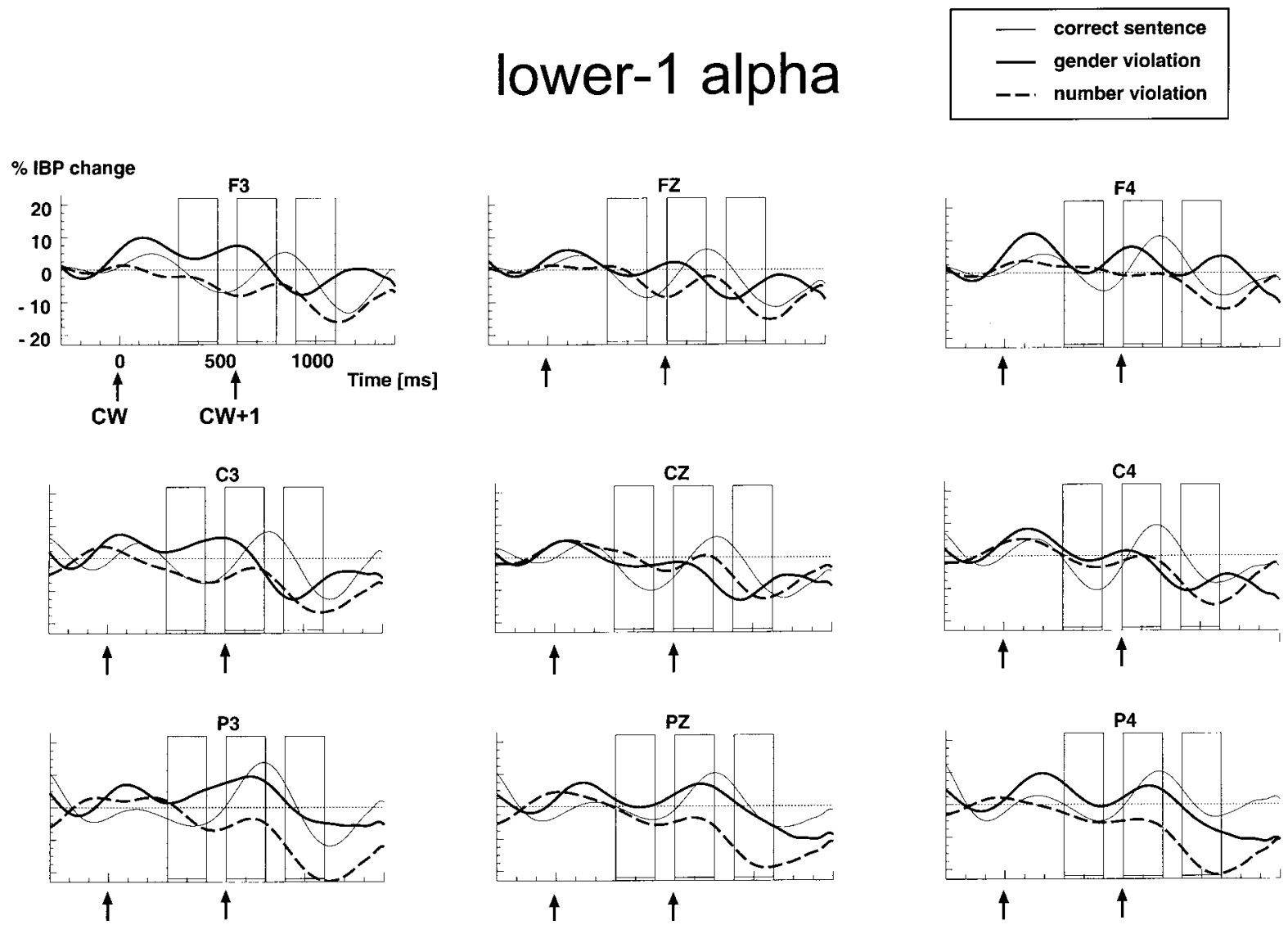

FIG. 4. Time course of IBP changes in the lower-1 $\alpha$ frequency band, at selected electrodes. See legend to Fig. 2 for further details.

changes during sentence processing (Bastiaansen et al., 2002): a window of 300-500 ms after onset of CW, which corresponds to the interval of maximal IBP change in the previous study, and two subsequent 200 ms intervals (600-800 and 900-1100 ms post-CW) which were primarily included in order to verify to which extent the differences found in the first interval persist over time. Note that the three intervals also correspond to the usual latencies of Ianguage-related ERP components, respectively the $\mathrm{N} 400$ and the LAN; the early part of the P600/SPS component; and the average latency of a late positivity. ${ }^{1}$

In order to assess whether the IBP changes differ among conditions, ANOVAs for repeated measurements were performed separately for each of the five frequency bands and each of the time intervals. For each ANOVA, factors were condition (correct, gender violation, number violation), anteriority (anterior, posterior), and hemisphere (left, right). Significant inter-

\footnotetext{
${ }^{1}$ In agreement with existing literature (e.g., Hagoort and Brown, 2000) an ERP analysis of the present data (Van Berkum et al., 2000) revealed a biphasic positivity, with the early part corresponding to the P600/SPS and having a significantly more anterior distribution than the late positivity.
}

actions were clarified by breaking them down into simple effect, or by formulating post hoc contrasts. Where necessary, degrees of freedom were corrected using the Greenhouse-Geisser $\varepsilon$; in these cases corrected P values are reported throughout Results.

\section{RESULTS}

Grand average percentages IBP changes for the five different frequency bands are presented in Figs. 2-6, for a representative selection of el ectrodes. The results of the statistical analyses are presented separately below for each time window.

\section{0- to 500-ms Interval}

The ANOVAs on the IBP changes in the lower-1, lower-2, and upper $\alpha$ frequency bands show that the power in this time interval does not differ significantly from zero for any of these bands (all $P$ values $>0.3$ ). Furthermore, in these bands there are no significant effects of the within-subjects factors. In the $\delta$ band, a significant power increase was present $\left(\mathrm{F}_{1,17}=39.41\right.$, $\mathrm{P}$ $<0.001$ ), with a right anterior maximum (interaction of anteriority with hemisphere: $F_{1,17}=7.74, P=0.013$ ). 


\section{lower-2 alpha}
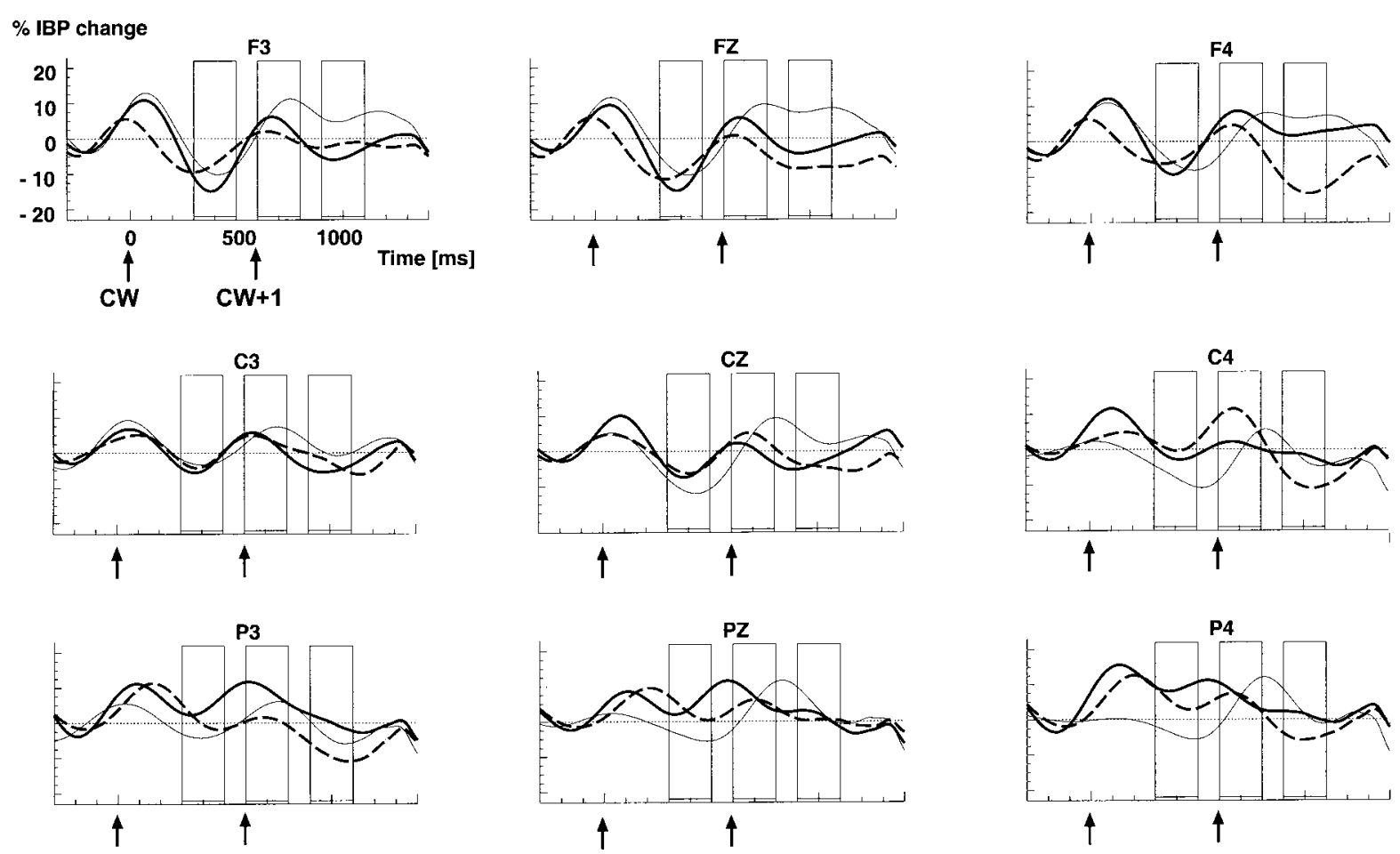

FIG. 5. Time course of IBP changes in the lower- $2 \alpha$ frequency band, at selected electrodes. See legend to Fig. 2 for further details.

There were no effects of condition in this band. The most interesting results occur in the $\theta$ frequency band. Here we observed a power increase of $7.05 \%$ in the time interval of 300-500 ms after word onset $\left(F_{1,17}=8.47\right.$, $\mathrm{P}=0.010$ ). F urthermore, $\theta$ power increases are larger over the anterior part of the scalp than over posterior sites: 9.9 vs. $4.2 \% \theta$ power increase, respectively (main effect of anteriority: $\left.F_{1,17}=4.32, P=0.026\right)$. A planned comparison between correct sentences on the one hand, and gender and number agreement violations on the other hand, shows that $\theta$ power increases are significantly larger for sentences containing violations $\left(F_{1,17}\right.$ $=5.12, \mathrm{P}=0.019 ; 2.5 \%$ vs $13.6 \% \theta$ power increase). Interestingly, the scalp distribution of the $\theta$ power increase differs among conditions (interaction of condition with hemisphere: $F_{2,34}=9.56, P=0.01$ ). Simple effect tests of hemisphere for each condition showed that, in the correct sentences, $\theta$ power increases are bilaterally symmetrical $\left(F_{1,17}=0.04, P=0.95\right)$. In the gender violation condition, a right-hemisphere dominance is found $\left(F_{1,17}=9.98, P=0.003\right)$, while in the number violation condition, $\theta$ power is largest over the left hemisphere $\left(F_{1,17}=8.34, P=0.005\right)$. The differences in scalp topography of the IBP changes in the $\theta$ frequency band are graphically represented in Fig. 7.

\section{0- to $800-m s$ I nterval}

In this time interval, power changes differed significantly from zero only in the $\delta$ band $\left(\mathrm{F}_{1,17}=12.36, \mathrm{P}=\right.$ 0.03 , pointing to a power increase in this band). Significant within-subjects effects were found only in the $\theta$ band. In this band, the power is largest over the right hemisphere in the gender violation condition (see Fig. 3). This produces an interaction of condition with hemisphere $\left(\mathrm{F}_{2,34}=4.93, \mathrm{P}=0.007\right.$; simple effect of condition in the left hemisphere: $F_{2,34}=1.03 ; P=0.191$; in the right hemisphere: $\left.F_{2,34}=2.57 ; P=0.050\right)$. This effect additionally produces a main effect of hemisphere $\left(F_{1,17}=13.59 ; P=0.001\right)$.

\section{0- to 1100-ms Interval}

In this time interval, power changes differed from zero only in the lower- $1 \alpha$ band $\left(\mathrm{F}_{1,17}=4.58\right.$; $\left.\mathrm{P}=0.024\right)$, in the sense that there was, on average, a power decrease of $6.59 \%$. There were no significant within-subjects factors in this frequency band.

In the $\delta$ band, a main effect of hemisphere $\left(\mathrm{F}_{1,17}=\right.$ $9.42, \mathrm{P}=0.007$ ) together with an interaction of hemisphere with anteriority $\left(\mathrm{F}_{1,17}=8.37, \mathrm{P}=0.01\right)$ indicate that here the power is largest over right anterior sites. 


\section{upper alpha}
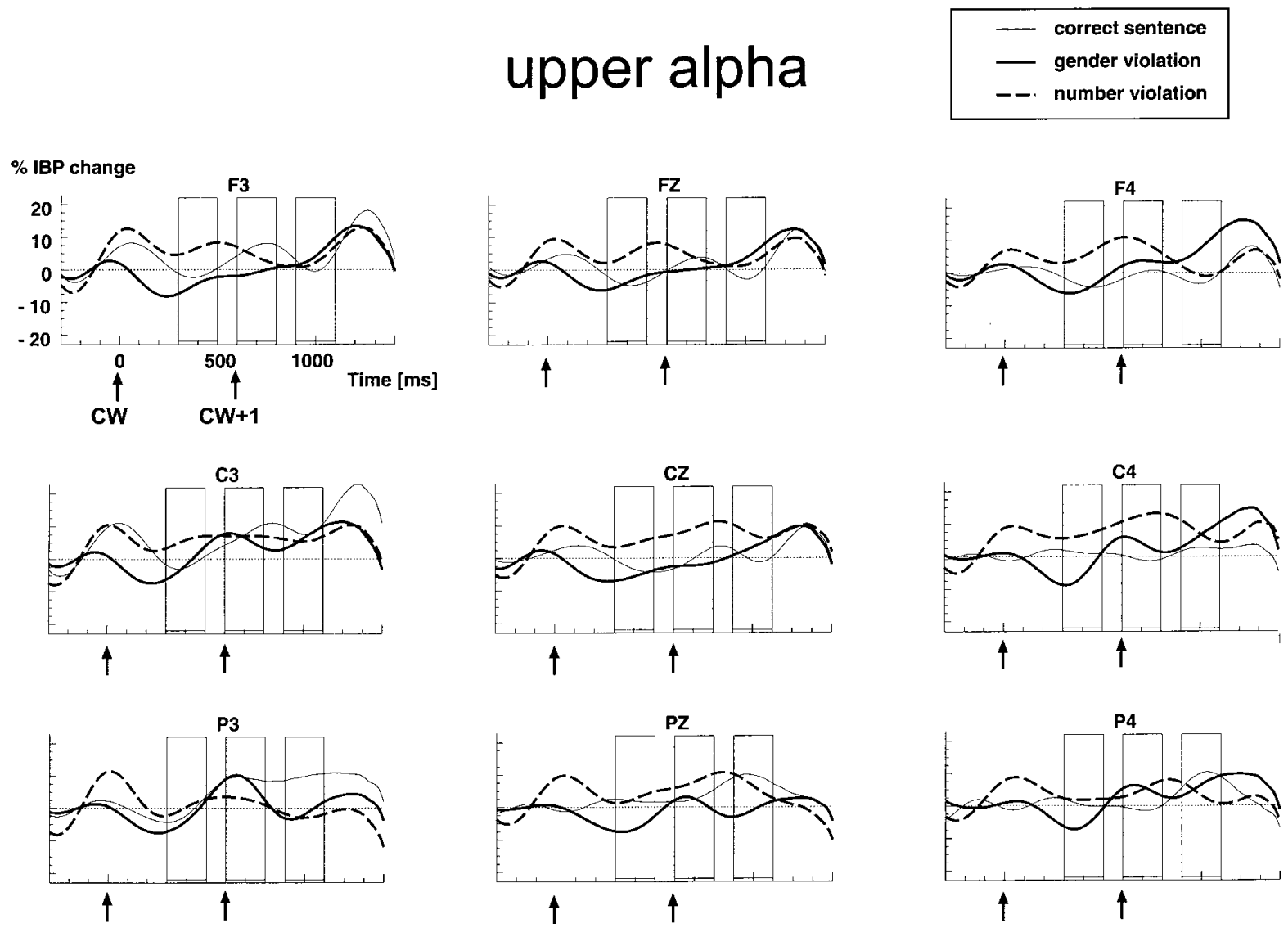

FIG. 6. Time course of IBP changes in the upper $\alpha$ frequency band, at selected electrodes. See legend to Fig. 2 for further details.

In the analysis on $\theta$ power, a main effect of hemisphere $\left(\mathrm{F}_{1,17}=4.01 ; \mathrm{P}=0.030\right)$ reveals that in this time window, $\theta$ power is largest over the right hemisphere. Visual inspection of Fig. 3 suggests that, just as for the 600- to $800-\mathrm{ms}$ interval, this effect is caused by larger power over the right hemisphere in the gender violation condition. However, this is not supported by the statistical analyses, since the interaction of condition with hemisphere fails to reach significance $\left(\mathrm{F}_{2,34}=\right.$ 1.71; $\mathrm{P}=0.104)$.

In the lower- $2 \alpha$ band, an interaction of condition with hemisphere $\left(F_{2,34}=2.74, P=0.042\right)$ seems to indicate that over the right hemisphere in the number violation condition the power values are smaller than in the other conditions. Furthermore, a marginally significant condition $*$ hemisphere $*$ anteriority $\left(\mathrm{F}_{2,34}=\right.$ $1.98, P=0.076$ ) seems to indicate that this difference tends to be larger over anterior sites. However, all the underlying simple effects and contrasts are nonsignificant (all $P$ values $>0.5$ ), making the above-mentioned interaction difficult to interpret. Finally, there were no significant effects in the analysis of the upper $\alpha$ band.

In order to further illustrate the frequency specificity of the reported effects, we computed, for one represen- tative subject, average power spectra based on the EEG data in the baseline interval of the correct sentence condition and in the 300- to 500-ms intervals of all three conditions. Figure 8 shows the relevant difference spectra (i.e., subtractions of two power spectra) in each condition for this subject. The figure confirms the results of the statistical analyses, in that general word presentation effects are most prominent in the $\delta$ and $\theta$ frequency ranges, whereas syntactic violation effects are restricted to (or at least most prominent in) the $\theta$ frequency range. Note that we did not compute grand average power spectra (i.e., averaged over all subjects), because that would result in a smearing of the individually adjusted frequency bands, so Fig. 8 serves an illustrative purpose only.

\section{DISCUSSION}

In order to explore whether the analysis of induced band power changes in the $\delta, \theta$, and $\alpha$ frequency bands of the EEG is sensitive to the syntactic parsing operations involved in language comprehension, subjects read sentences that were either correct or contained syntactic (number or gender agreement) violations. The presentation of the critical words elicited a signif- 
30

$\%$

IBP

Correct

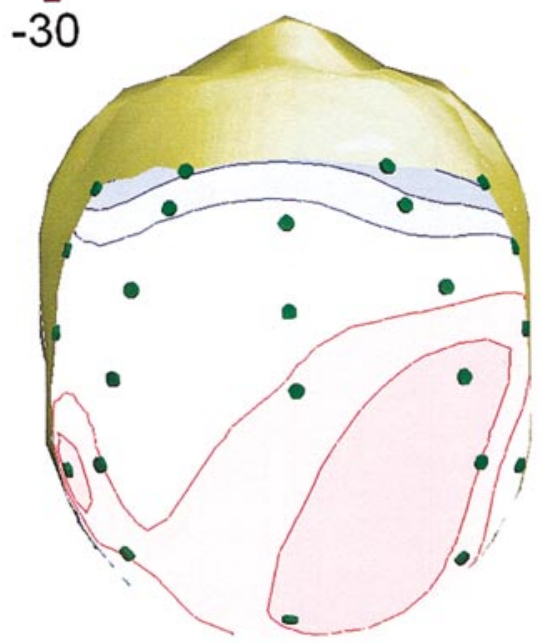

Gender

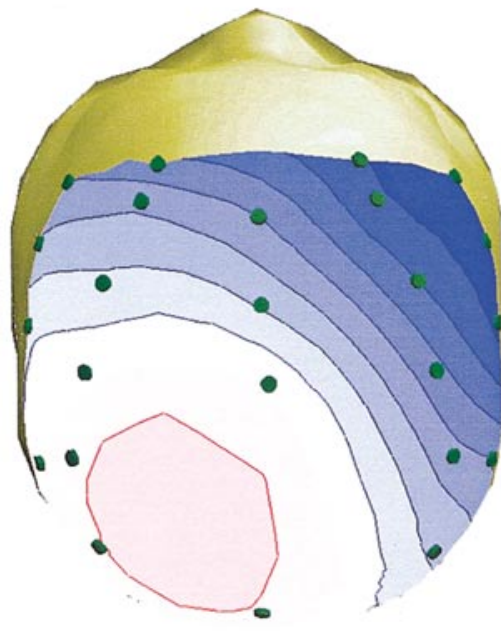

Number

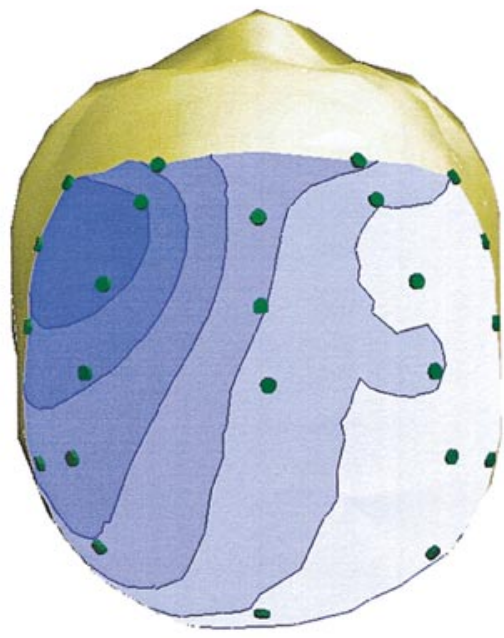

\section{difference spectra, subject 02}

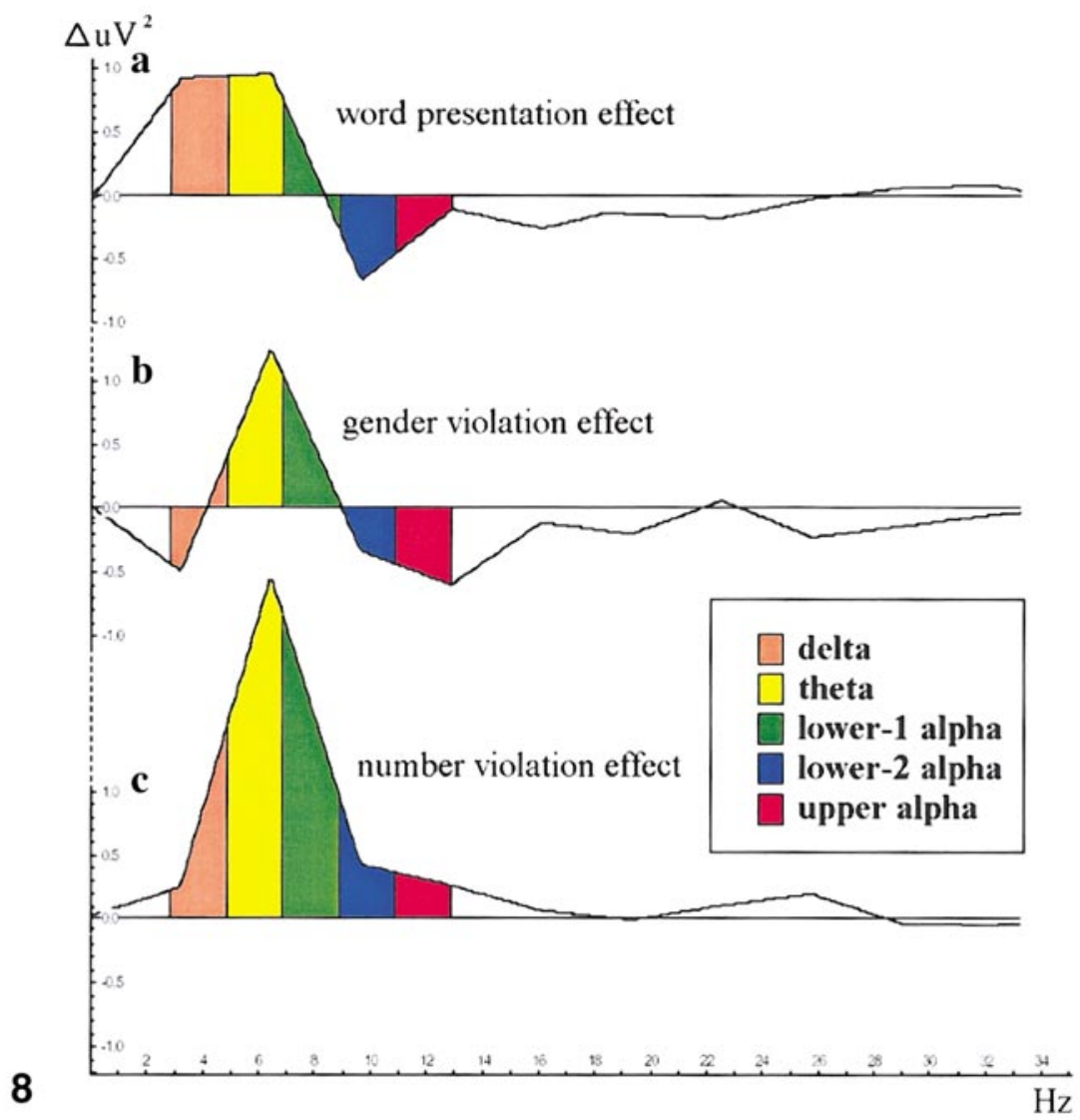

FIG. 7. Topographical distribution of the $\theta$ power increase in the interval of $300-500 \mathrm{~ms}$ after word onset, in an orthogonal projection onto a realistically shaped head model. Blue denotes a power increase, red denotes a power decrease (in \% IBP). Spacing of isopercentage lines is $3 \%$ IBP change. Scaling as indicated. Note the clear differences in scalp topography among the three conditions. 
icant increase in power in the $\delta$ and $\theta$ frequency bands, in an interval of 300-500 ms after word onset, which was largest over anterior parts of the scalp. Such a statistically significant power increase was not observed in the higher (i.e., various $\alpha$ ) frequency bands. Most interestingly, only in the $\theta$ band did we observe reliable differences between correct sentences and sentences containing a violation (that is, except for an intricate interaction of condition with hemisphere in the lower- $2 \alpha$ band in the interval of 900-1100 ms after onset of the critical word, which we considered uninterpretable). The $\theta$ power increase was larger following words that constituted a violation than following correct words. In addition, different types of violations led to different scalp distributions of the $\theta$ power increase. While normal sentences elicited bilaterally symmetrical $\theta$ power increases at frontal electrodes, number agreement violations were followed by $\theta$ power increases with a left-frontal maximum, and gender agreement violations led to $\theta$ power increases with a right-frontal maximum. Furthermore, the gender violation effect outlasted the number violation effect, the former being significant also in the intervals of 600800 and 900-1100 ms after the onset of the critical words. In sum, we found both quantitative and qualitative differences between correct sentences and sentences containing violations.

As a first remark, it should be noted that irrespective of sentence context, each word elicited a phasic power increase in the $\theta$ frequency band, roughly between 300 and $500 \mathrm{~ms}$ after word onset. This is consistent with the findings of our earlier analysis in which we studied the responses to words in correct sentences (Bastiaansen et al., 2002). In that analysis words were followed by a highly similar $\theta$ power increase. Roughly the same effect was found also in a recent, as yet unpublished experiment performed in our laboratory, using different stimuli and different subjects. Thus, the phasic $\theta$ power increase 300-500 ms following word onset appears to be a robust phenomenon. Note that in the present study we found also an increase in the $\delta$ frequency band. However, it is likely that a part of what we defined as $\delta$ in the present study is essentially the low end of an oscillatory phenomenon in the $\theta$ frequency range. This is supported by the power spectra in Fig. 8. Findings supporting this notion have also been reported by Klimesch et al. (2000). This leaves the question of how to interpret the $\theta$ response following word presentation. Does it truly reflect an increase in oscillatory activity, or can it be explained otherwise? First, one may argue that in order to demonstrate that power changes reflect oscillatory phenomena, one must identify a corresponding peak in the power spectrum. However, in our opinion this is too restrictive a criterion for establishing oscillatory activity. A peak in the power spectrum is present only if an oscillatory component dominates the EEG (as is often the case for the $\alpha$ rhythm). However, there can be oscillatory activity which is hidden in the "noise" of other (rhythmic or nonrhythmic) activities, in a similar way as an ERP component is hidden in the noise of single-trial EEG data. Therefore, the prerequisite of a spectral peak is in most cases too conservative. Next, it may be argued that power increases are a reflection of broadband power changes and that the fact that we find an increase in $\theta$ power is merely an artifact (i.e., a result of the filtering procedure). However, this interpretation can be excluded on the basis of the high frequency specificity of our findings, as indicated by the statistical analyses, and supported by the difference spectra presented in Fig. 8, which clearly show that power increases are maximal in the $\theta$ frequency range. Thus, we feel that an increase in power in a circumscribed, narrow frequency range cannot be interpreted otherwise than in terms of an increase in oscillatory activity.

Recall that an increase in power of oscillatory activity is thought to reflect an increase in synchrony of the oscillating firing patterns of local neuronal populations. F urthermore, the latency of the present $\theta$ power increase, i.e., 300-500 ms poststimulus, roughly falls in the range of well-known ERP components related to language processing, such as the N400 and the LAN. Therefore, a possible interpretation of such a phenomenon is that it reflects the activation of a local functionally coherent network, related to some aspect of word processing, either lexical or in relation to sentence (or even discourse) context.

The most important observation of the present experiment is that the $\theta$ power increase is larger in response to words constituting syntactic violations than to words in a correct sentence context. In addition,

FIG. 8. Subtraction of power spectra in different conditions, for a representative subject (s02), at electrodes where the reported effects are most prominent (i.e., electrode Fz for a, F4 for b, and F 3 for c). Color coding indicates the definition of frequency bands for this subject, based on his individual $\alpha$ peak (i.e., $10.9 \mathrm{~Hz}$ ). (a) Subtraction of power spectrum computed in the 300- to 500-ms interval following the CW ( $\mathrm{CW}$ interval) in the correct sentence condition from that computed in the corresponding baseline interval. Note the power increase in the $\delta$ and $\theta$ frequency bands and the decrease in the higher frequency bands, which are a result of word presentation. (b) Subtraction of power spectrum in the $\mathrm{CW}$ interval of the gender violation condition from the power spectrum in the CW interval of the correct condition. N ote the highly selective power increase in the $\theta$ frequency band resulting from the gender violation. The relative power decrease in the $\alpha$ bands is not significant over subjects. (c) Subtraction of power spectrum in the CW interval of the number violation condition from the power spectrum in the CW interval of the correct condition. Only the relative increase in $\theta$ power is significant over subjects. 
whereas number and gender violations elicited clear qualitative differences in scalp topography of the $\theta$ response reported here, and ERP analysis of the same data revealed only quantitative differences between the two violation types (the P600/SPS was larger in the number than in the gender violation condition, but the ERP morphologies and scalp topographies were identical, cf., Van Berkum et al., 2000).

The most important question that arises regards the specific functional interpretation of the qualitatively different $\theta$ increases following different types of syntactic violations. One interpretation of the effect would be that it reflects oscillatory dynamics involved in syntactic parsing operations, associated with the initial detection of a parsing problem and/or its processing consequences. Another interpretation, which extends beyond the domain of strictly syntactic processing, may be that the differential $\theta$ response to gender and number violations reflects the fact that whereas (in the language used here) the former constitutes a purely syntactic violation, the latter additionally leads to a conceptual-semantic problem: in sentences like "I saw several dark cloud on the horizon" it is unclear whether one or several clouds are meant. Studying $\theta$ responses to purely semantic violations in sentences may be helpful in further delineating whether such an interpretation of the present effect is viable. Finally, an entirely nonlinguistic interpretation can certainly not be excluded. For instance, it may well be that the occurrence of a syntactic violation is encoded in episodic memory as an unusual event and that it is this episodic encoding which is reflected in the larger $\theta$ increase following violations compared to the control words. Such an interpretation would be in line with numerous findings relating $\theta$ power increases to episodic encoding processes (e.g., Klimesch, 1999). Qualitatively different effects following different types of syntactic violations could then reflect an interaction between encoding and the type of process that signals the violation.

An intriguing result of the present study is that syntactic manipulations yield highly frequency-specific effects, in the sense that they induced increases in oscillatory activity in the $\theta$ frequency band only. N ote that this frequency specificity has also repeatedly been reported by Klimesch and co-workers, during episodic memory tasks (see Klimesch, 1999, for a review). The question is then, why are the syntax-related IBP differences restricted to the $\theta$ frequency band? At this point, we can only speculate about an answer to this question.

Since we are dealing here with $\theta$ activity that appears over frontal electrodes, it might be tempting to conclude that our results are related to a phenomenon termed frontal midline $\theta$. This oscillatory activity is usually thought to originate in the anterior cingulate cortex, in medial frontal cortical areas, or in the lateral sensorimotor cortex (e.g., Gevins et al., 1997; Asada et al., 1999; Luu and Tucker, 2001). Frontal midline $\theta$ usually increases in power during the execution of demanding tasks (e.g., Sasaki et al., 1996; Gevins et al., 1997; McE voy et al., 2001). I ts modulation appears to be independent of the type of tasks, and therefore frontal midline $\theta$ has been mostly related to domaingeneral processes such as directing sustained, focused attention (Gevins et al., 1997; I shii et al., 1999) or action monitoring and evaluation (Luu and Tucker, 2001). In this context, it is conceivable that the present $\theta$ effect is related to error monitoring (a syntactic violation is obviously an error). The fact that the errorrelated negativity (ERN, cf., Falkenstein et al., 2000) is also thought to originate from the anterior cingulate cortex, e.g. (Dehaene et al., 1994; Holroyd and Coles, 1998), would support this idea. The error-monitoring interpretation of the present $\theta$ effect could be investigated by studying $\theta$ reactivity during tasks which are known to elicit an ERN. It should be noted, however, that it is difficult to explain the different scalp distributions for gender and number violations with a mere error-monitoring interpretation.

Alternatively, the present results may be tentatively linked to corticolimbic dynamics. The changes in $\theta$ power that we record at the scalp may (at least partially) be brought about under the influence of the hippocampal system. This proposition, based on Miller's (1991) theory of corticohippocampal interplay, is worked out in detail elsewhere (Bastiaansen and $\mathrm{Ha}-$ goort, in press); therefore, we will only briefly address it here. The reasoning starts from a number of wellknown facts. First, the human hippocampal system (meant here to include the hippocampus proper, the dentate gyrus and the subiculum, and the peri- and entorhinal and parahippocampal cortices), generates theta activity. ${ }^{2}$ Second, there is evidence that the hippocampal system is involved in language processing: Next to an increasing number of fMRI studies that demonstrate activation of the hippocampal system during tasks in which linguistic stimulus material is used (e.g., Daselaar et al., 2001; Crinion et al., 2001; Crosson et al., 2001; Taylor et al., 2001; Keller et al., 2001; Gleissner and Elger, 2001), local field potentials directly recorded from the medial temporal lobe (most probably from regions of the hippocampal system) have been found to be highly sensitive to relatively subtle linguistic manipulations, such as semantic congruence

\footnotetext{
${ }^{2}$ Although in the past there has been considerable debate as to whether hippocampal $\theta$ activity occurs exclusively in lower mammals or also in humans (e.g., Steward and F ox, 1990; Lopes da Silva, 1991; Miller, 1991), it has been repeatedly demonstrated, with the help of appropriate spectral analysis techniques, that the human hippocampus is able to generate $\theta$ rhythms (e.g., Halgren et al., 1978, 1985; Arnolds et al., 1980; Zaveri et al., 2001).
} 
of words within a sentence, and the presentation of open-class vs cl osed-class words (McCarthy et al., 1995; Nobre and McCarthy, 1995). Third, there are massive connections between the hippocampal system and almost every part of the neocortex. These connections, based on average estimates of axonal conduction times in the rat, have total conduction times for one hippocampocorticohippocampal loop of 120 to $200 \mathrm{~ms}$. This time interval roughly corresponds to one period of $\theta$ (Miller, 1991). As a result, patterns of resonant loops may emerge between the cortex and the hippocampal system, which are strengthened, through Hebbian processes, by the precise timing (namely, at $\theta$ frequencies) of cells firing at the hippocampal end of the loop. In Miller's (1991) view, one of the major functions of this corticohippocampal interplay is that each set of loops will synchronously activate functionally related, but widely dispersed cell groups in the neocortex, which by themselves have insufficient mutual interconnections to be able to form a cell assembly by the usual Hebbian principles. Thus, in this view the hippocampal system would play an orchestrating role in synchronizing the activity of neuronal populations at its preferred frequency, i.e., $\theta$.

Although in our opinion this hypothesis is certainly appealing, it is highly speculative. Moreover, defacto it cannot be empirically evaluated with scalp EEG recordings, since such recordings only provide information about the firing of cortical neurons. One would have to assume that $\theta$ power reactivity in the scalp EEG uniquely, or at least to a large extent, reflects processes at the cortical end of a hippocampocortical loop, an assumption that can obviously not be made without empirical support. Therefore, an empirical evaluation of this assumption would be highly desirable. In this context, studies combining intracranial recordings from the hippocampal system with scalp recordings would be of interest, since such studies could directly address the question of the relationship between hippocampal $\theta$ and scalp-recorded $\theta$. In summary, although the generators of the observed $\theta$ modulations are as yet unknown, at least they seem to result in a relatively specific modulation of its scalpsurface characteristics.

\section{REFERENCES}

Arnolds, D. E. A. T., Lopes da Silva, F. H., Aitink, J . W., Kamp, A., and Boeijinga, P. 1980. The spectral properties of hippocampal EEG related to behavior in man. Electroenceph. Clin. Neurophysiol. 50: 324-328.

Asada, H., Fukuda, Y., Tsunoda, S., Yamaguchi, M., and Tonoike, M. 1999. Frontal midline theta rhythms reflect alternative activation of prefrontal cortex and anterior cinmgulate cortex in humans. Neurosci. Lett. 274: 29-32.

Bastiaansen, M. C. M., and Hagoort, P. 2002. Event-induced theta responses as a window on the dynamics of memory. Cortex, in press.
Bastiaansen, M. C. M., Van Berkum, J . J . A., and Hagoort, P. 2002. Event-related theta power increases in the human EEG during online sentence processing. Neurosci. Lett. 323: 13-16.

Berger, H. 1929. Über das Elektroencephalogramm des Menschen. Arch. Psychiat. Nerv-Krankh. 87: 527-570.

Brown, C., and Hagoort, P 1999. The Neurocognition of Language Processing. Oxford Univ. Press, New York.

Brown, C., and Hagoort, P. 2000. On the electrophysiology of Ianguage comprehension: Implications for the human language system. In Architectures and Mechanisms for Language Processing (M. W. Crocker, M. Pickering, and C. Clifton J r. Eds.), pp. 213237, Cambridge University, Cambridge, UK.

Crinion, J ., Blank, S. C., and Wise, R. 2001. Centyral neural systems for both narrative speech comprehension and propositional speech production. Neurol mage 13: S520.

Crosson, B., Benefield, H., Cato, M. A., Sadek, J . R., Gopinath, K., Soltysik, D., Bauer, R. M., Auerbach, E. J ., Gokcay, D., Leonard, C. M., and Briggs, R. W. 2001. Left parahippocampal gyrus activity during language generation. Neurol mage 13: S521.

Daselaar, S. M., Rombouts, S. A. R. B., Veltman, D. J ., Raaijmakers, J . G. W., Lazeron, R. H. C., and J onker, C. 2001. Parahippocampal activation during successful recognition of words: a self-paced event-related fMRI study. Neurol mage 13: 1113-1120.

Dehaene, S., Posner, M. I., and Tucker, D. M. 1994. Localisation of a neural system for error detection and compensation. Psychol. Sci. 5: 303-305.

Falkenstein, M., Hoormann, J ., Christ, S., and Hohnsbein, J . 2000. ERP components on reaction errors and their functional significance: A tutorial. Biol. Psychol. 51: 87-107.

Friederici, A. D., Hahne, A., and Mecklinger, A. 1996. Temporal structure of syntactic parsing: Early and late event-related brain potential effects. J . Exp Psychol. Learning Memory Cognition 22: 1219-1248.

Fries, P., Neuenschwander, S., Engel, A. K., Goebel, R., and Singer, W. 2001. Rapid feature selective neuronal synchronization through correlated latency shifting. Nat. Neurosci. 4: 194-200.

Gevins, A., Smith, M. E., McEvoy, L., and Yu, D. 1997. High-resolution EEG mapping of cortical activation related to working memory: Effects of task difficulty, type of processing, and practise. Cereb. Cortex 7: 374-385.

Gleissner, U., and Elger, C. E. 2001. The hippocampal contribution to verbal fluency in patients with temporal lobe epilepsy. Cortex 37: 55- 63.

Gunter, T. C., Friederici, A. D., and Schriefers, H. 2000. Syntactic gender and semantic expectancy: ERPs reveal early autonomy and late interaction. J . Cogn. Neurosci. 12: 556-568.

Hagoort, P., and Brown, C. M. 1999. Gender electrified: ERP evidence on the syntactic nature of gender processing. J . Psycholing. Res. 28: 715-728.

Hagoort, P., and Brown, C. M. 2000. ERP effects of listening to speech compared to reading: The P600/SPS to syntactic violations in spoken sentences and rapid serial visual presentation. Neuropsychologia 38: 1531-1549.

Hagoort, P., Brown, C. M., and Groothusen, J . 1993. The syntactic positive shift (SPS) as an index of syntactic processing. Lang. Cogn. Proc. 8: 439-483.

Hagoort, P., Brown, C. M., and Osterhout, L. 1999. The neurocognition of syntactic processing. In TheNeurocognition of Language( $P$. Hagoort and C. M. Brown, Eds.), pp. 273-318. Oxford Univ. Press, Oxford.

Halgren, E., Babb, T. L., and Crandall, P. H. 1978. Human hippocampal formation EEG desynchronizes during attentiveness 
and movement. Electroencephalogr. Clin. Neurophysiol. 44: 778781 ,

Halgren, E., Smith, M. E., and Stapleton, J . M. 1985. Hippocampal field-potentials evoked by repeated vs. non-repeated words. In Electrical Activity of the Archicortex (G. Buszaki and C. H. Vanderwolf, Eds.), pp. 67-81. Akademiai Kiadó, Budapest.

Holroyd, C. B., Dien, J ., and Coles, M. G. 1998. Error-related scalp potentials elicited by hand and foot movements: Evidence for an output-independent error-processing system in humans. Neurosci. Lett. 242: 65- 68.

Ishii, R., Shinisaki, K., Ukai, S., Inouye, T., Ishihara, T., Y oshimine, T., Hirabuki, N., Asada, H., Kihara, T., Robinson, S. E., and Takeda, M. 1999. Medial prefrontal cortex generates frontal midline theta rhyhtm. Neuroreport 10: 675- 679.

Kahana, M. J ., Sekuler, R., Caplan, J . B., Kirschen, M., and Madsen, J. R. 1999. Human theta oscillations exhibit task dependence during virtual maze navigation. Nature 399: 781-784.

Kalcher, J., and Pfurtscheller, G. 1995. Discrimination between phase-locked and non-phase-locked event-related EEG activity. Electroenceph. Clin. Neurophysiol. 94: 381-384.

Keller, S., Downes, J . J ., Mackay, C., Westwell, N., Baker, G., and Roberts, N. 2001. Language ability and hippocampal integrity. Neurol mage 13: S549.

Klimesch, W. 1999. EEG alpha and theta oscillations reflect cognitive and memory performance: A review and analysis. Brain Res. Rev. 29: 169-195.

Klimesch, W., Doppelmayr, M., Schwaiger, J ., Winkler, T., and Gruber, W. 2000. Theta oscillations and the ERP old/new effect: Independent phenomena? Clin. Neurophysiol. 111: 781-793.

Knosche, T. R., and Bastiaansen, M. C. M. 2002. On the time resolution of event-related desynchronization: A simulation study. Clin. Neurophysiol. 113(5): 754-763.

Kutas, M., and Hillyard, S. A. 1980. Reading senseless sentences: Brain potentials reflect semantic incongruity. Science 207: 203205.

Kutas, M., and Van Petten, C. 1995. Psycholinguistics electrified: event-related brain potential investigations. In Handbook of Psycholinguistics (M. Gernsbacher, Ed.), pp. 83-143. Academic Press, San Diego.

Lachaux, J . P., Rodriguez, E., Martinerie, J ., and Varela, F. J . 1999. Measuring phase synchrony in brain signals. Hum. Brain Mapp. 8: 194-208.

Lachaux, J. P., Rodriguez, E., Le Van Quyen, M., Lutz, A., Martinerie, J., and Varela, F. J. 2000. Studying single trials of phase synchronous activity in the brain. Int. J . Bifurc. Chaos 10: 24292439.

Lisman, J . E., and I diart, M. A. P. 1995. Storage of $7 \pm 2$ short-term memories in oscillatory subcycles. Science 267: 1512-1515.

Lopes da Silva, F. H. 1991. Hippocampal RSA in humans. Trends Neurosci 14: 138.

Lopes da Silva, F. H., and Pfurtscheller, G. 1999. EEG synchronization and desynchronization. In Event-Related Desynchronization, Handbook of Electroencephalography and Clinical Neurophysiology (G. Pfurtscheller and F. Lopez da Silva, Eds.), revised series, Vol. 6, pp. 3-11. Elsevier, Amsterdam.

Luu, P., and Tucker, D. M. 2001. Regulating action: Alternating activation of midline frontal and motor cortical networks. Clin. Neurophysiol. 112: 1295-1306.
Martin, A., and Chao, L. L. 2001. Semantic memory and the brain: Structure and processes. Curr. Opin. Neurobiol. 11: 194-201.

McCarthy, G., Nobre, A., Bentin, S., and Spencer, D. D. 1995. Language-related field potentials in the anterior-medial temporal lobe: I. Intracranial distribution and neural generators. J . Neurosci. 15: 1080-1089.

McClelland, J. L., St J ohn, M., and Taraban, R. 1989. Sentence comprehension: A parallel distributed processing approach. Lang. Cogn. Proc. 4: 287-335.

McEvoy, L. K., Pelloucoud, E., Smith, M. E., and Gevins, A. 2001. Neurophysiological signals of working memory in normal aging. Cogn. Brain Res. 11: 363-376.

Miller, R. 1991. Cortico-hippocampal Interplay and the Representation of Contexts in the Brain. Springer-Verlag, Berlin.

Nobre, A., and McCarthy, G. 1995. Language-related filed potentials in the anterior-medial temporal lobe: II. Effects of word type and semantic priming. J . Neurosci. 15: 1090-1098.

Nunez, P. L. 1995. Neocortical Dynamics and Human EEG Rhythms. Oxford Univ. Press, New York.

Nunez, P. L., Srinivasan, R., Wetdorp, A. F., Wijesinghe, R. S., Tucker, D. M., Silberstein, R. B., and Cadusch, P. J . 1997. EEG coherency I: Statistics, reference electrode, volume conduction, Laplacians, cortical imaging and interpretation at multiple scales. Electroenceph. Clin. Neurophysiol. 103: 499-515.

Osterhout L., and Holcomb, P. J . 1992. Event-related brain potentials elicited by syntactic anomaly. J . Mem. Lang. 31: 785- 608.

Osterhout, L., and M obley, L. A. 1995. Event-related brain potentials elicited by failure to agree. J. Mem. Lang. 34: 739-773.

Sasaki, K., Tsujimoto, T., Nishikawa, S., Nishitani, N., and I shihara, T. 1996. Frontal mental theta wave recorded simultaneously with magnetoencephalography and electroencephalography. Neurosci. Res. 26: 79-81.

Singer, W. 1993. Synchronization of cortical activity and its putative role in information processing and learning. Ann. Rev. Physiol. 55: 349-374.

Steward, M., and Fox, S. E. 1990. Do septal neurons pace the hippocampal theta rhythm? Trends Neurosci. 13: 163-168.

Tallon-Baudry, C., and Bertrand, O. 1999. Oscillatory gamma activity in humans and its role in object representation. Trends Cogn. Sci. 3: 151-162.

Taylor, S. J ., McAndrews, M. P., Makarec, K., and Mikulis, D. 2001. Event-related fMRI reveals a synchronized hippocampal system associated with verbal encoding. Neurol mage 13: S747.

Treves, A., and Rolls, E. T. 1994. Computational analysis of the role of the hippocampus in memory. Hippocampus 4: 374-391.

Van Berkum, J. J . A., Brown, C. M., and Hagoort, P. 1999a. Early referential context effects in sentence processing: Evidence from event-related brain potentials. J . Mem. Lang. 41: 147-182.

Van Berkum, J. J . A., Brown, C. M., and Hagoort, P. 1999b. When does gender constrain parsing? Evidence from ERPS. J . Psycholing. Res. 28(5): 555-571.

Van Berkum, J . J . A., Zwitserlood, P., Brown, C. M., and Hagoort, P. 2000. Processing gender and number agreement in parsing: An ERP-based comparison. In Proceedings of the 6th Conference on Architectures and Mechanisms of Language Processing (AMLaP2000).

Zaveri, H. P., Dockrow, R. B., De Lanerolle, N. C., and Spencer, S. 2001. Distinguishing subtypes of temporal lobe epilepsy with background hippocampal activity. Epilepsia 42: 725-730. 